\title{
Effect of Continuum Scattering on Early-type Supergiants Spectra
}

\author{
Nikoghossian A.* \\ NAS RA V.Ambartsumian Byurakan Astrophysical Observatory, Byurakan 0213, Aragatsotn Province, Armenia
}

\begin{abstract}
The effect of radiation scattering in continuum on the frequency distribution of the observed flux in atmospheres of supergiants of the late B and A spectral classes is treated. Thomson scattering on free electrons, which is important for hydrodynamic balance and wind dynamics in extended atmospheres of these stars, is considered as a specific scattering mechanism in the continuum. It is shown how stars of the same bolometric luminosity and with equal radii can belong to different spectral classes. The dependence of the continuum shortwave drift on the density of rarefied plasma has been established. The role of Thomson scattering in different domains of the hydrogen spectrum is revealed.
\end{abstract}

\section{Introduction}

An important place among early-type supergiants is belonging to stars of spectral class A and its adjacent subclasses of types B and $\mathrm{F}$. These stars are visually brightest in our own galaxy, as well as in external galaxies. There is evidence for mass outflows and extending envelops in all of these objects, As a part of luminous variable stars they are characterised by comparatively quiescent states interrupted from times to times by outbursts. The shells accelerating by radiation pressure form a kind of more or less opaque winds with moderate terminal velocity (Humphreys \& Davidson, 1979, Owocki et al., 2004).

It is well established that scattering of radiation in the continuum plays an important role in forming spectra of these objects. Among other of possible mechanisms of scattering, we will treat the Thomson scattering on free electrons. The high temperature rarefied plasma with highly ionized hydrogen and partly helium evidently makes this mechanism dominant.

The understanding of the importance of taking scattering into account in the continuum goes back to pioneering work of Schuster (1905). The mechanism of Thomson scattering on free electrons in the context of atmospheres of early-type stars was discussed by Ambartsumian (1938) who found that the color temperature of such stars may differ from effective temperature. He concluded that taking the latter instead of the former may lead to the undervalues in estimating the radii of such stars. For temperatures of about $10^{5} \mathrm{~K}$ he got the upper limit for the gas density below which the electron scattering effect becomes important.

The extreme non-stationarity of luminous and massive stars manifests itself not only in variability of their spectral class but also in specific changes in temperature, emission and absorption, spectrum discontinuities, line profiles. The detailed and comprehensive study of these phenomena will obviously provide a better understanding of the evolution of the stars under study at a later post-core H-burning stages.

In this connection, there is an urgent need in detailed study of electron scattering effect on the main characteristics of the observed spectrum. In this paper we treat the influence of Tomson scattering on the light curve and and its discontinuities in the hydrogen spectrum. The paper can be regarded as a generalization of results we obtained previously in Nikoghossian \& Israelian (1996).

*nikoghoss@yahoo.com 


\section{The frequency distribution of the flux of radiation emerging from a grey atmosphere.}

We start with considering the effect of the scattering process in continuum on the frequency distribution of the observed spectrum. It should be noted that, in order to clarify this question, the scattering mechanism itself is not essential, although it is Thomson scattering on free electrons that is the implication in this paper because of its importance in studying the spectra of early-type supergiants. The equation of radiation transfer for this problem has a form

$$
\eta \frac{\mathrm{d} I_{\nu}}{\mathrm{d} r}=-\left(\alpha_{\nu}+\sigma\right) I_{\nu}+\sigma J_{\nu}+\alpha_{\nu} B_{\nu}(T),
$$

where the following notations are used: $\alpha_{\nu}$ is the coefficient of absorption in the continuum and $\sigma$ is the coefficient of the continuous scattering, each per unit length; $B_{\nu}(T)$ is the Planck function and

$$
J_{\nu}=\oint I_{\nu} \frac{d \omega}{4 \pi} .
$$

The natural boundary condition for the problem at issue is $I(0, \eta)=0$ for $\eta>0$.

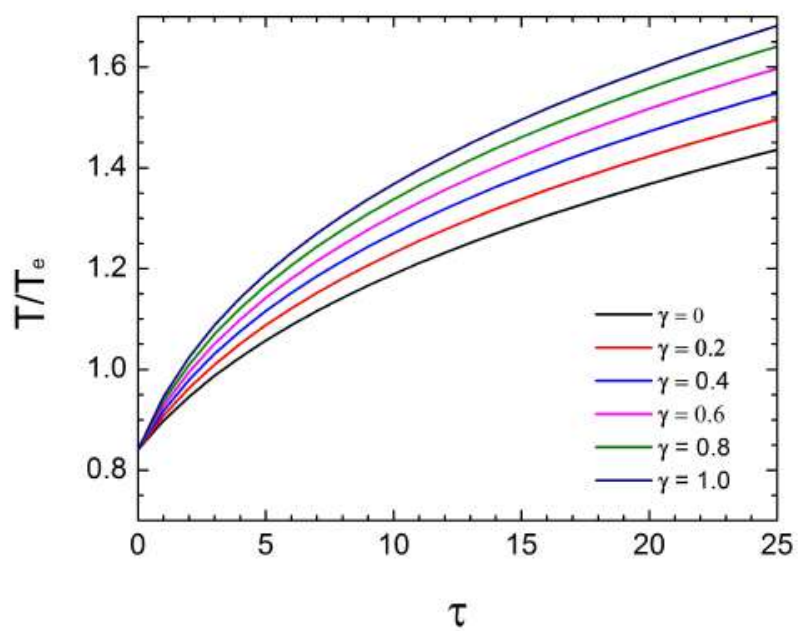

Figure 1. The temperature gradients dependence on the level of electron scattering.

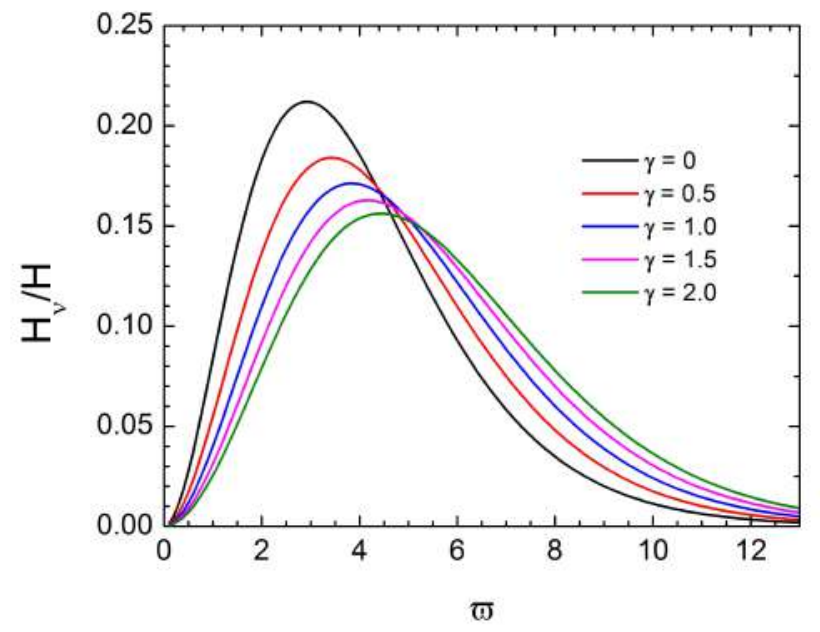

Figure 2. The drift of the continuum due to electron scattering.

It is well known Mihalas (1970) that the scattering processes do not change the form of the condition of radiative equilibrium since the proper emitted and absorbed terms are canceled, so that 
we have

$$
\int_{0}^{\infty} \alpha_{\nu} J_{\nu} \mathrm{d} \nu=\int_{0}^{\infty} \alpha_{\nu} B_{\nu}(T) \mathrm{d} \nu .
$$

Let us now consider a gray hydrogen semi-infinite atmosphere, with no radiation incident on its boundary. As an averaged coefficient of absorption we take Planck's mean given by (see e.g.,Sobolev 1960, Mihalas 1970)

$$
\bar{\alpha}=\frac{\int_{0}^{\infty} \alpha_{\nu} B_{\nu}(T) \mathrm{d} \nu}{\int_{0}^{\infty} B_{\nu}(T) \mathrm{d} \nu} .
$$

Now replacing $\alpha_{\nu}$ with $\bar{\alpha}$ and integrating Eq,(1) over all the spectrum we can write

$$
\eta \frac{\mathrm{d} I}{\mathrm{~d} \tau}=(1+\gamma) I-\gamma J-B(T)
$$

where $d \tau=-\bar{\alpha} d r, \gamma=\sigma / \bar{\alpha}$ and

$$
J=\int_{0}^{\infty} J_{\nu} \mathrm{d} \nu, \quad B(T)=\int_{0}^{\infty} B_{\nu}(T) \mathrm{d} \nu .
$$

Now the condition of radiative equilibrium (3) can be rewritten as

$$
J=B(T)=\frac{a c}{4 \pi} T^{4}
$$

where $a$ is the Stefan constant.

Having this in mind, Eq.(5) takes a form

$$
\eta \frac{\mathrm{d} I}{\mathrm{~d} t}=I-B(T) .
$$

and the problem is reduced to the solution of the classical Milne equation for the function $J(t)$, where $t=(1+\gamma) \tau$

$$
J(t)=\frac{1}{2} \int_{0}^{\infty} J\left(t^{\prime}\right) \operatorname{Ei}\left(\left|t-t^{\prime}\right|\right) \mathrm{d} t^{\prime}
$$

with well-known solution

$$
J(t)=\pi F(t+q(t))
$$

where $q(t)$ is Hopf's function. In view of $\mathrm{Eq}(6)$ we find for the temperature gradient in the medium

$$
T(t)=T_{\text {eff }}(t+q(t))^{1 / 4}
$$

Thus, we arrive at the temperature gradient of the common functional form where, however, the opacity is due to both the pure absorption and the scattering process.

Fig.1 demonstrates the run of temperature with optical depth in the atmosphere in terms of $\tau$. As would be expected, the Thomson coherent scattering process acting on quanta of all wavelengths across the spectrum increases the opacity hindering the escape of quanta from the medium. This obviously leads to steeper rise in temperature within the medium. It is important to note that this occurs under constant values of effective temperature (as well as of surfaces temperature, in the Eddington approximation) and integral flux. This, in its turn, implies that the star with the generated scattering in its outer layers will not change the luminosity. At the same time the observer should obviously detect a change in the star's spectrum towards short waves, as it is shown in Fig. 2 that presents the theoretical fluxes versus $\gamma$ given in the Eddington approximation 


$$
\frac{H_{\nu}(0)}{H}=0.31 \varpi^{3} \int_{0}^{\infty} \frac{\mathrm{E}_{2}(\tau) \mathrm{d} \tau}{\mathrm{e}^{\varpi p(\tau, \gamma)}-1}
$$

where $\varpi=\frac{h \nu}{k T_{*}}, p(\tau, \gamma)=\frac{3}{4}(1+\gamma) \tau+\frac{1}{2}$ This implies that the spectral class of the star moves to the earlier classes without any change in the luminosity. On the HR diagram it looks as a drift to the left of it parallel to the axis of spectral classes.

$$
L=4 \pi R_{*}^{2} \sigma T_{\mathrm{eff}}^{4}
$$

is replaced by the color temperature. This obviously leads to the diminished values for radii of stars. We point now to another possible errors that can arise in using the $L$ versus $T_{\text {eff }}$ Hertzsprung-Rassel diagram for this kind of peculiar stars. Indeed, having at our disposal the luminosity and the radius of a star determined by the other independent methods, the spectral class predicted by the H-R diagram may differ from the actual one.

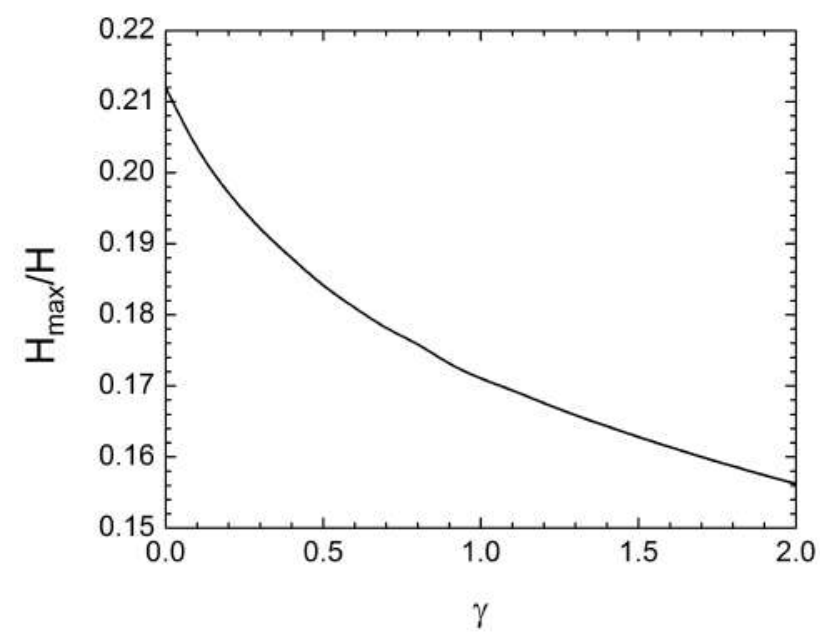

Figure 3. Variation of the maximum value of the flux with an increase of the level of the electron scattering.

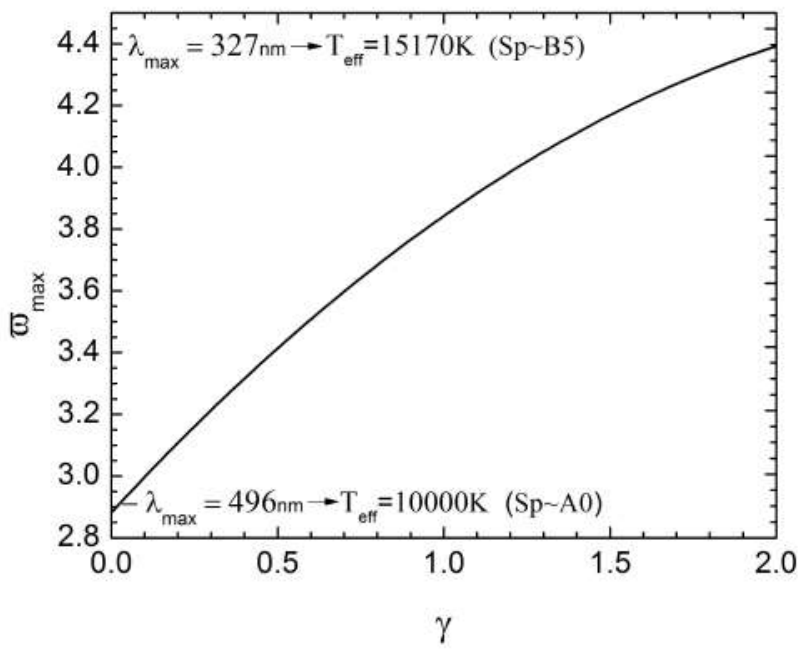

Figure 4. Drift in the location of the maximum flux due to the electron scattering.

Let us now turn to the question of under which conditions the Thomson scattering effect becomes important. Ambartsumian (1938) was the first who considered this point for the helium spectrum in 
the context of atmospheres of the W-R type stars by taking $T=10^{5} \mathrm{~K}$. Employing the formulas for the mean absorption coefficient given in (Chandrasekhar, 1934) and (Unsöld, 1934) (he obtained for the upper limit for the gas density $\rho \leq 10^{-9} \mathrm{~g} / \mathrm{cm}^{3}$ which corresponds to the ions or electrons number density of the order $\sim 10^{15} \mathrm{~cm}^{-3}$. Similar estimate for the hydrogen spectrum with $T=10^{4} \mathrm{~K}$ we obtain is $\rho \leq 10^{-13} \mathrm{~g} / \mathrm{cm}^{3}$ and correspondingly, $n_{\mathrm{e}}=\sim 10^{11} \mathrm{~cm}^{-3}$.

However, it is also of great interest the effect of continuum scattering in different domains of the spectrum. To this end, let us consider the hydrogen spectrum where absorption coefficient per unit length is given by the well-known formula (see, e,g,, Sobolev, 1963)

$$
\alpha_{\nu}=C(T) \frac{n_{e} n^{+}}{\nu^{3}}\left(\frac{2 \chi_{1}}{k T} \sum_{i=i_{0}}^{\infty} \frac{1}{i^{3}} \mathrm{e}^{-\frac{\chi_{i}}{k T}}+1\right)\left(1-e^{-\frac{h \nu}{k T}}\right),
$$

where the Gaunt coefficients are taken equal to 1,

$$
C(T)=\frac{2^{4} \pi^{2} e^{6} k T}{3 \sqrt{3} \operatorname{ch}(2 \pi m k T)^{3 / 2}},
$$

and for other values, well-known designations have been adopted: $h$ and $k$ are correspondingly Planck's and Boltsman's constants, $e$ and $m$ are the charge and the mass of electron. The value of $i_{0}$ is determined by inequality $h \nu \geq \chi_{i}$. For $T=10^{4} K$ we obtain

$$
\alpha_{\nu}=36.71 \cdot 10^{5} \frac{n_{e} n^{+}}{\nu^{3}}\left[31.44\left(7.05 \cdot 10^{6}+6.44+0.21+\ldots\right)+1\right]\left(1-e^{-\frac{h \nu}{k T}}\right), .
$$

We are interested in the first greatest three terms in parentheses corresponding to the Lyman, Balmer and Pashen series. To this end, we find it expedient to use some averaged approximate values of absorption coefficients for each of these domains in the shortwave vicinity of the series jumps. Representing these estimates in the form $\alpha_{\nu}=A \cdot n_{e} n^{+} \mathrm{cm}^{-1}$, we find $A=2.3 \cdot 10^{-32}, 1.4 \cdot 10^{-36}, 5.4 \cdot 10^{-37}$ correspondingly for the Lyman, Balmer and Pashen jumps. On the other hand, the Thomson scattering coefficient $\sigma=n_{e} \sigma_{0}$, where

$$
\sigma_{0}=\frac{8 \pi}{3}\left(\frac{e^{2}}{m c^{2}}\right)^{2}
$$

is of the order of $10^{-25} \mathrm{~cm}^{2}$, Then assuming that hydrogen is completely ionized, we get $\sigma / \alpha_{\nu}=$ $2,9 \cdot 10^{7} n_{e}^{-1}$ in the vicinity of the Lyman jump and, respectively, $4,8 \cdot 10^{11} n_{e}^{-1}, 1,2 \cdot 10^{12} n_{e}^{-1}$ for the Balmer and Pashen jumps. Thus, we see that the effect begins to affect the rarified plasma starting from the electron densities of the order of $10^{12} \mathrm{~cm}^{-3}$ and less which in the case of the fully ionized hydrogen corresponds to the matter density of the order of $10^{-12} \mathrm{~g} / \mathrm{cm}^{3}$. This result is consistent with that obtained by Ambartsumian for temperatures of the order of $10^{5} \mathrm{~K}$ in connection of the W-R stars. At the same time, we see that the electron scattering effect begins to appear primarily in the long-wave domains of the spectrum. For instance, the effect in the UV domain may become significant only at densities less than $10^{-6}, 10^{-7} \mathrm{~g} / \mathrm{cm}^{3}$

\section{Effect of Thomson scattering on spectrum discontinuities}

Our goal in this section is to determine the effect of multiple coherent scattering on free electrons on the Lyman, Balmer and Pashen discontinuities in the hydrogen spectrum. To this end, let us turn to transfer equation Eq.(1) in the Eddington approximation. We have

$$
\frac{1}{3} \frac{\mathrm{d} J_{\nu}}{\mathrm{d} \tau}=\left(k_{\nu}+\gamma\right) \bar{H}_{\tau}, \frac{\mathrm{d} \bar{H}_{\nu}}{\mathrm{d} \tau}=k_{\nu}\left(J_{\nu}-B_{\tau}(T)\right),
$$

where, again, as above, $d \tau=-\bar{\alpha} d r, k_{\nu}=\alpha_{\nu} / \bar{\alpha}$ and $\bar{H}_{\nu}=H_{\nu} / 2 \pi$. The set of equations Eq.(18) can be reduced to

$$
\frac{\mathrm{d}^{2} J_{\nu}}{\mathrm{d} \tau^{2}}=\bar{\kappa}_{\nu}^{2}\left(J_{\nu}-B_{\nu}(T)\right),
$$




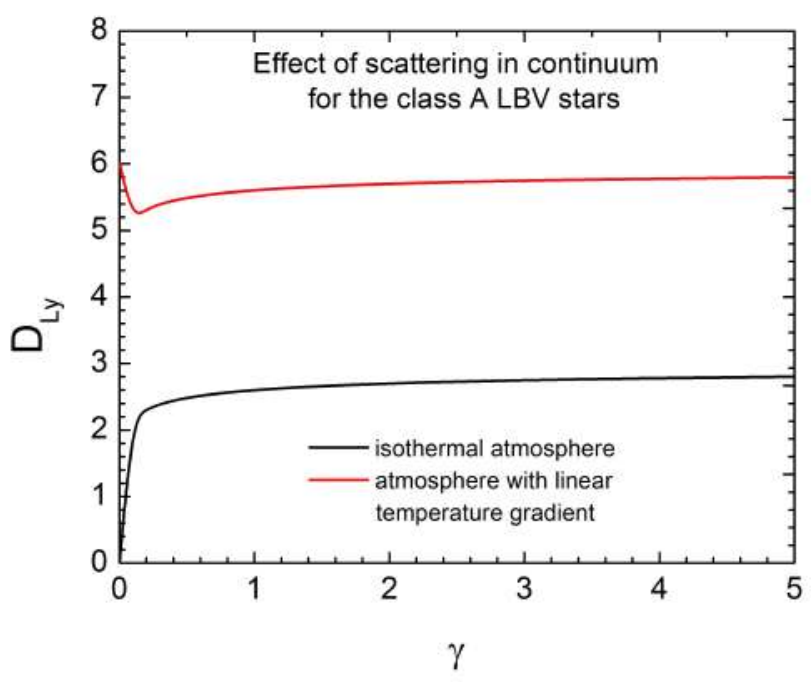

Figure 5. Effect of Thomson scattering on Lyman discontinuities.

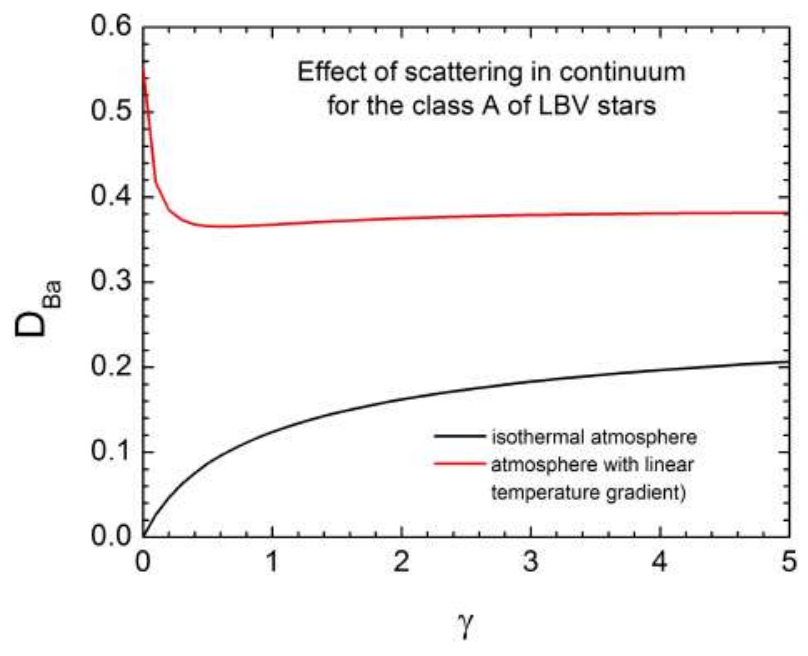

Figure 6. The same as in Fig.5 for Balmer discontinuities.

with $\bar{\kappa}_{\nu}^{2}=3 k_{\nu}\left(k_{\nu}+\gamma\right)$.

In solving Eq.(19) we assume that the depth variation of temperature is linear so that $B_{\nu}(\tau)=$ $B_{\nu}\left(T_{0}\right) \cdot\left(1+\beta_{\nu} \tau\right)$, where (see, e.g., Sobolev 1960)

$$
\beta_{\nu}=\frac{3}{8} \frac{h \nu}{k T_{0}}\left(1-\exp \left(-\frac{h \nu}{k T_{0}}\right)\right)^{-1} .
$$

Eq.(19) is solved under usual assumptions of absence of external incident radiation and requiring the solution to be bounded at infinitely great depths. We obtain

$$
\bar{H}_{\nu}(0)=B_{\nu}\left(T_{0}\right)\left(1+\frac{\beta_{\nu}}{\bar{\kappa}_{\nu}}\right) /\left(2+\sqrt{3\left(1+\frac{\sigma}{\alpha_{\nu}}\right)}\right)
$$

This allows to find the requisite values of discontinuities $H_{\nu>\nu_{0}} / H_{\nu<\nu_{0}}$, where $H_{\nu<\nu_{0}}$ and $H_{\nu>\nu_{0}}$ are emergent fluxes before and after jumps. Knowledge of $\alpha_{\nu<\nu_{0}} / \bar{\alpha}$ and $\alpha_{\nu>\nu_{0}} / \bar{\alpha}$ from Eq,(16) leads to the required result.

In the next part of this study we will examine some other manifestations of electron scattering in the context of the spectra of early type supergiants.

Figures 5-7 demonstrate dependence of Lyman, Balmer and Pashen discontinuities $D=\log \frac{H_{\nu>\nu_{0}}}{H_{\nu<\nu_{0}}}$ on the relative role of Thomson scattering $\gamma$. Each of the figures depicts two curves related to the 


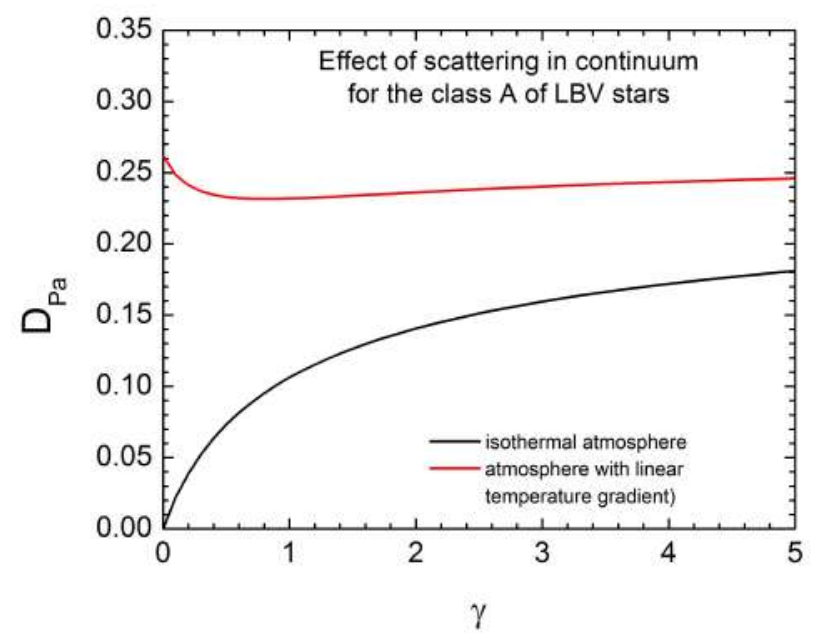

Figure 7. The same as in Figs.5,6 for Pashen discontinuities.

isothermal atmosphere (black) and to the atmosphere with linear temperature gradient (red). The given curves allow making a number of important conclusions. As it should be expected, the jumps formed in the atmosphere with the depth-varying temperature are greater as compared to those in the isothermal atmosphere.Further, the scattering process in the isothermal atmosphere leads to jumps due to the fact that quanta with a frequency before and after jumps come out of different depths and the number of scatterings they undergo before leaving the atmosphere is different. With increasing role of scattering the quanta that leave the outer layers of the atmosphere are, naturally, more affected, so the size of the jumps tends to increase with increasing $\gamma$. This effect is similar to the behaviour of the absorption line profiles in the nucleus and wings with an increase in the probability of the quantum's re-radiation at an elementary scattering act. A more complex picture is observed in the case of an atmosphere with a temperature gradient, where jumps are formed in the absence of scattering. Here, the behaviour of the jump size with $\gamma$ depends on the relative role of true absorption and Thomson scattering processes in different parts of the spectrum.

\section{References}

Ambartsumian V. A., 1938, TsAGI Uchenye Zapiski, 22, 19

Chandrasekhar S., 1934, Zeitschrift für Astrophysik, 8, 167

Humphreys R. M., Davidson K., 1979, ApJ, 232, 409

Mihalas D., 1970, Stellar atmospheres

Nikoghossian A., Israelian G., 1996, JQSRT, 56, 501

Owocki S. P., Gayley K. G., Shaviv N. J., 2004, ApJ, 616, 525

Schuster A., 1905, ApJ, 21, 258

Unsöld A., 1934, Zeitschrift für Astrophysik, 8, 32 